\title{
Effects of MHD slow shocks propagating along magnetic flux tubes in a dipole magnetic field
}

\author{
N. V. Erkaev $^{1}$, V. A. Shaidurov ${ }^{2}$, V. S. Semenov ${ }^{3}$, and H. K. Biernat ${ }^{4,5}$ \\ ${ }^{1}$ Institute of Computational Modelling, Russian Academy of Sciences, Krasnoyarsk-36, 660036, Russia \\ ${ }^{2}$ State University of Krasnoyarsk, 660041, Krasnoyarsk, Russia \\ ${ }^{3}$ Institute of Physics, State University of St. Petersburg, St. Petergof, 198504, Russia \\ ${ }^{4}$ Space Research Institute, Austrian Academy of Sciences, Schmiedlstrasse 6, 8042 Graz, Austria \\ 5 also at: Institute of Geophysics, Astrophysics, and Meteorology, University of Graz, 8010 Graz, Austria
}

Received: 7 August 2001 - Revised: 11 February 2002 - Accepted: 28 February 2002

\begin{abstract}
Variations of the plasma pressure in a magnetic flux tube can produce MHD waves evolving into shocks. In the case of a low plasma beta, plasma pressure pulses in the magnetic flux tube generate MHD slow shocks propagating along the tube. For converging magnetic field lines, such as in a dipole magnetic field, the cross section of the magnetic flux tube decreases enormously with increasing magnetic field strength. In such a case, the propagation of MHD waves along magnetic flux tubes is rather different from that in the case of uniform magnetic fields. In this paper, the propagation of MHD slow shocks is studied numerically using the ideal MHD equations in an approximation suitable for a thin magnetic flux tube with a low plasma beta. The results obtained in the numerical study show that the jumps in the plasma parameters at the MHD slow shock increase greatly while the shock is propagating in the narrowing magnetic flux tube. The results are applied to the case of the interaction between Jupiter and its satellite Io, the latter being considered as a source of plasma pressure pulses.
\end{abstract}

\section{Introduction}

Magnetohydrodynamics (MHD) has proved to be a useful approach commonly used for modeling of space plasma behavior in many aspects. However, in gerneral, MHD's problems are rather complicated to solve, in particular, in cases of a nonsteady, three-dimensional plasma flow. A possible way to simplify the problem is to use asymptotic methods based on series expansion on small parameters. Models of thin magnetic flux tubes are examples of such an approach, where the thickness of the magnetic tube is considered to be much smaller than the length scale of the tube (e.g. the curvature radius). Different types of such models exist based on a thin magnetic flux tube approximation. The first type is a

Correspondence to: $\mathrm{H}$. K. Biernat

(helfried.biernat@oeaw.ac.at) model applicable for the Sun (Fisher et al., 2000), where the magnetic field consists of separate, thin magnetic flux tubes that accumulate magnetic energy generated by magnetic field stretching. Inside such tubes, the plasma pressure is quite small with respect to the magnetic pressure. Another type is a model of magnetic flux tubes with an enhanced plasma pressure. These models are relevant for cases of plasma perturbations in the magnetospheres of the Earth and other planets. For these models, the behavior of plasma and the shape of the magnetic tube are strongly dependent on the ratio of plasma and magnetic pressures, which is called the plasma beta $(\beta)$. In cases of large plasma beta (or $\beta \sim 1$ ), the shape of the magnetic flux tube depends strongly on the distribution of plasma pressure inside the tube. The latter can be relevant to magnetic tubes near a reconnecting magnetopause or in the magnetotail plasma sheet (Chen and Wolf, 1999). In the internal parts of the magnetosphere, the magnetic field is quite strong and the plasma beta is likely to be small. This plasma beta can be considered as an additional small parameter. In such a case, a small plasma pressure has no great influence on the shape of the magnetic tube. This means that a configuration of the magnetic flux tube can be considered to be undisturbed. The latter is the case corresponding to the problem studied in the present paper.

The aim of our paper is to model the propagation of MHD slow waves which are produced by nonsteady plasma pressure variations, along a dipole magnetic flux tube. The magnetic tube thickness, normalized to the curvature radius of the tube, as well as the plasma beta are considered to be small parameters.

It is important to note that a local pressure enhancement produces mainly slow magnetosonic waves rather than Alfvén waves due to of the low plasma beta (see Appendix). Another consequence of the low plasma beta is that the local disturbances of the shape of the magnetic tube can be considered to be very small, since the plasma pressure is much less than the magnetic pressure.

It is an important fact that the slow mode wave propa- 
gates inside a dipole flux tube with a progressively decreasing cross section. As a result, the wave amplitude (velocity maximum) has to increase along the flux tube rather than decrease. For linear waves, this effect is qualitatively explained in the Appendix. For nonlinear waves, this effect is studied numerically in our calculations.

In principle, the proposed model can be used for the analysis of wave processes occurring inside the magnetospheres of the Earth and other planets, where different reasons for the initial pressure pulses might exist. These pressure pulses can be related to the reconnection pulses occurring at the magnetopause or at the neutral sheet of the magnetotail. For example, a pressure pulse in the Earth's magnetosphere can be produced by the deceleration of a fast plasma flow caused by a reconnection pulse in the magnetotail. Plasma related to the reconnected magnetic flux tube first starts to move rapidly along the plasma sheet towards Earth, and then it is decelerated in the increasing geomagnetic field.

In particular, we apply this model to the interaction between Jupiter and its satellite Io, which is considered to be a source of pressure enhancements (Combi et al., 1998). There exists a direct observation of the plasma pressure in the vicinity of Io (Frank et al., 1996). According to this observation, the pressure has two peaks with an enhancement factor of $\sim 3$. However, between these peaks, the spacecraft seems to cross the cold ionosphere of Io, and thus, the data points are not valid for the warm plasma in the torus. Extrapolating the data points with a Gaussian function reveals that the real enhancement of the plasma pressure must be even bigger, $\sim 6$. This is the reason why we use in our calculations the initial pressure amplitudes $P_{1} / P_{0}=3$ and $P_{1} / P_{0}=6\left(P_{1}\right.$ is the pressure maximum, and $P_{0}$ is the background pressure at the point of the pressure maximum).

In this case, the magnetic flux tube can be considered as "thin", since the cross size of the tube $\sim R_{i}\left(R_{i}\right.$ is the Io radius) is much smaller than the curvature radius of the tube $\sim 5 R_{J}$ ( $R_{J}$ is the Jupiter radius).

\section{Basic equations}

To describe nonsteady wave perturbations of magnetic field and plasma parameters, we apply the system of ideal MHD equations (Landau and Lifshitz, 1960)

$\rho \frac{\partial \boldsymbol{V}}{\partial t}+\rho(\boldsymbol{V} \cdot \nabla) \boldsymbol{V}+\nabla \Pi-\frac{1}{\mu_{0}}(\boldsymbol{B} \cdot \nabla) \boldsymbol{B}=\rho \boldsymbol{F}$,

$\frac{\partial \rho}{\partial t}+\operatorname{div}(\rho \boldsymbol{V})=0$

$\frac{\partial}{\partial t}\left(\frac{P}{\rho^{\kappa}}\right)+(\boldsymbol{V} \cdot \nabla)\left(\frac{P}{\rho^{\kappa}}\right)=0$,

$\frac{\partial \boldsymbol{B}}{\partial t}-\operatorname{rot}(\boldsymbol{V} \times \boldsymbol{B})=0, \quad \operatorname{div} \boldsymbol{B}=0$.

Here, $\rho, \boldsymbol{V}, P, \boldsymbol{B}$ are mass density, bulk velocity, plasma pressure and magnetic field, respectively. The external force

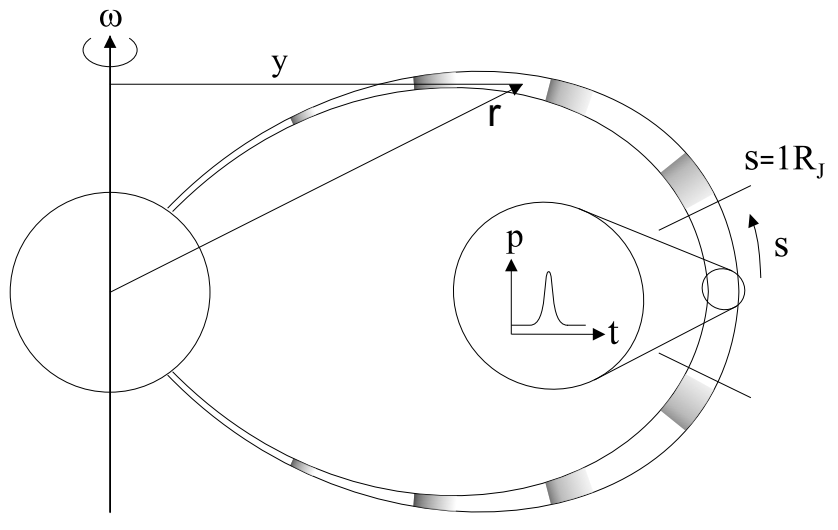

Fig. 1. Schematic illustration of the development of a nonlinear slow mode wave due to a pressure pulse. The parameter $S$ is introduced as the distance measured along the flux tube.

$\boldsymbol{F}$ is a sum of centrifugal and gravitation forces referred to a mass unit

$\boldsymbol{F}=\nabla\left(\frac{G M}{r}+\frac{1}{2} \omega^{2} y^{2}\right)$,

where $G$ is the gravitational constant, $M$ is the mass of the planet, $r$ is the radial distance from the center of the planet, $y$ is the distance to the rotational axis, and $\omega$ is the angular speed, respectively. Quantity $\Pi$ denotes the total pressure (the sum of magnetic and plasma pressures), $\Pi=P+B^{2} /\left(2 \mu_{0}\right)$, and $\kappa$ is the polytropic exponent.

From the mathematical point of view, we have a nonsteady initial-boundary problem for the ideal MHD equations. The geometrical situation of the problem is illustrated in Fig. 1. The ionospheres of Io and Jupiter with non-ideal plasmas are not included in our calculation domain. More specifically, we consider the ideal MHD model to be suitable to describe Alfvén as well as magnetosonic waves propagating in the region between the ionospheres of Io and Jupiter.

For computational convenience, we normalize the magnetic field and the respective plasma parameters as follows

$$
\begin{gathered}
\tilde{\boldsymbol{R}}=\boldsymbol{R} / R_{J}, \quad \tilde{t}=t V_{A 0} / R_{J}, \quad \tilde{P}=P /\left(m_{i} N_{i} V_{A 0}^{2}\right), \\
\tilde{\rho}=\rho /\left(m_{i} N_{i}\right), \quad \tilde{\boldsymbol{B}}=\boldsymbol{B} / B_{0}, \tilde{\boldsymbol{V}}=\boldsymbol{V} / V_{A 0},
\end{gathered}
$$

where $R_{J}$ is the radius of the planet, subscript 0 denotes the initial background parameters corresponding to the starting point, $V_{A 0}$ is the Alfvén velocity, $m_{i}$ is the average ion mass, $N_{i}$ is the ion density, and $B_{0}$ is the magnetic field induction. For our calculations, we take two values of the initial plasma beta (at the equator of the tube): $\beta_{0}=0.02$ and $\beta_{0}=0.04$. The latter is relevant to the conditions at the Io orbit (Neubauer, 1998; Combi et al., 1998).

For the rotating plasma in the magnetic tube, the background plasma parameters are assumed to satisfy the equilibrium equation

$$
\frac{\partial P}{\partial S}=\frac{P}{k T} \frac{\partial}{\partial S}\left(\frac{G M m}{r}+0.5 m y^{2} \omega^{2}\right) .
$$


Here, $k$ is the Boltzmann constant, $T$ is the temperature assumed to be constant along the tube, $m$ is the average mass of particles. Eq. (6) describes a "hydrostatic" equilibrium in a rotating tube versus the curvilinear coordinate $S$. This behaviour of the background plasma density as it is used in our model is in agreement with the empirical model based on observations (Bagenal, 1994; Mei et al. 1995;)

We introduce the material coordinate $\alpha$, which is proportional to the mass of the plasma in the magnetic tube of the length $S$

$\alpha=\int_{0}^{S}(\rho / B) d S^{\prime}$.

This coordinate is a monotonic increasing function of the distance $S$ along the tube. In accordance with the mass conservation law, the quantity $\alpha$ is constant along the trajectory of a fluid element.

In addition to $\alpha$, we introduce two other coordinates, $\phi$ and $\psi$, which are the Euler potentials for the magnetic field

$\boldsymbol{B}=\nabla \phi \times \nabla \psi$.

It is evident from this equation that the coordinates $\phi$ and $\psi$ are constant along magnetic filed lines. As it is shown in the Appendix, the quantities $\alpha, \phi$, and $\psi$ are constant along trajectories of the fluid particles, and thus they can be interpreted as material coordinates.

Ideal MHD equations in material coordinates, known as "frozen-in", were analyzed by Pudovkin and Semenov (1977). Introducing the radius vector $\boldsymbol{r}$ as a function of $\alpha, \phi$, and $\psi$, we obtain the relations for the derivatives of the radius vector (for details, see the Appendix)

$$
\frac{\partial \boldsymbol{r}}{\partial \alpha}=\frac{\boldsymbol{B}}{\rho}, \quad \frac{\partial \boldsymbol{r}}{\partial t}=\boldsymbol{V}
$$

Using the material coordinates $\alpha, \phi$, and $\psi$, we transform (see Appendix) the ideal MHD equations in the normalized units into the following system

$$
\begin{aligned}
& \frac{\partial \boldsymbol{V}}{\partial t}-\frac{\partial \boldsymbol{B}}{\partial \alpha}+\frac{1}{\rho} \nabla \Pi=\boldsymbol{F}, \\
& P+\frac{1}{2}(\boldsymbol{B})^{2}=\Pi, \quad \frac{\partial}{\partial t}\left(\frac{\boldsymbol{B}}{\rho}\right)-\frac{\partial \boldsymbol{V}}{\partial \alpha}=0, \\
& \frac{\partial}{\partial t}\left(\frac{P}{\rho^{\kappa}}\right)=0, \quad \frac{D(x, y, z)}{D(\alpha, \phi, \psi)}=\frac{1}{\rho} .
\end{aligned}
$$

Here, $D(\ldots) / D(\ldots)$ denotes the Jacobian of the transformation. The components of the velocity and magnetic field can be obtained as derivatives of the radius vector with respect to $\alpha$ and $t$, (see Eq. 9). Equation (10) is the transformed MHD momentum equation, the two formulas in Eq. (11) are the expressions for the total pressure $\Pi$ and the transformed induction equation, and the two formulas in Eq. (12) express the polytropic law with exponent $\kappa$ and the mass conservation.
If the total pressure is a known function of the Cartesian coordinates, which does not depend explicitly on time, then the following energy equation is valid (see Appendix)

$$
\begin{aligned}
\frac{\partial}{\partial t}[ & \left.V^{2} / 2+B^{2} /(2 \rho)+P /((\kappa-1) \rho)+\Pi / \rho+\Phi\right] \\
& =\frac{\partial}{\partial \alpha}(V B) .
\end{aligned}
$$

Here, $\Phi$ is the sum of gravitational and centrifugal potentials. When the total pressure is a prescribed function of the Cartesian coordinates, the Eqs. $(10,11)$ are very similar to those for an elastic string, and Eq. (13) expresses the energy conservation for a string. Similar string equations were used by Erkaev and Mezentsev (1992) and Erkaev et al. (1996) for the magnetosheath region.

The total pressure can be considered to be a known function of $x, y$, and $z$ for thin layers or thin magnetic tubes due to the pressure balance across the layer. The total pressure inside the layer should be equal to the undisturbed total pressure outside the layer.

Assuming the initial background plasma beta $\beta_{0}$ to be small, we seek a solution as a series expansion with respect to this parameter

$$
\begin{aligned}
\boldsymbol{V}= & \sqrt{\beta_{0}}\left(\boldsymbol{V}^{(0)}(\tau, \alpha, \phi, \psi)+O\left(\beta_{0}\right)\right), \\
& \boldsymbol{B}=\boldsymbol{B}^{(0)}(\tau, \alpha, \phi, \psi)+O\left(\beta_{0}\right), \\
& \rho=\rho^{(0)}(\tau, \alpha, \phi, \psi)+O\left(\beta_{0}\right), \\
P= & \beta_{0} P^{(0)}(\tau, \alpha, \phi, \psi)+O\left(\beta_{0}^{2}\right), \quad t=\tau / \sqrt{\beta_{0}} .
\end{aligned}
$$

Using the expansion $(14,15)$ for the field-aligned velocity component, we obtain from system (9-12) the following equations

$\frac{\partial V^{(0)}}{\partial \tau}+\frac{1}{B^{(0)}} \frac{\partial P^{(0)}}{\partial \alpha}=F_{s}$,

$\frac{\partial}{\partial \tau}\left(\frac{B^{(0)}}{\rho^{(0)}}\right)-\frac{\partial V^{(0)}}{\partial \alpha}=0$,

$\frac{\partial S}{\partial \tau}-V^{(0)}=0$

$\frac{\partial}{\partial \tau}\left(\frac{P^{(0)}}{\rho^{(0)^{\kappa}}}\right)=0$.

Here, $F_{S}$ is the normalized force along the magnetic tube

$F_{s}=\frac{\partial}{\partial S}\left(\frac{G M \rho_{0}}{2 r P_{0}}+\frac{\omega^{2} y^{2} \rho_{0}}{4 P_{0}}\right)$

After simplifications based on a series expansion on $\beta_{0}$, the problem is reduced to that of one-dimensional, nonsteady equations with $\boldsymbol{V} \| \boldsymbol{B}$, which are solved numerically.

In our calculations, the energy Eq. (13), together with Eqs. (16) to (18), are used only for the first stage of the 


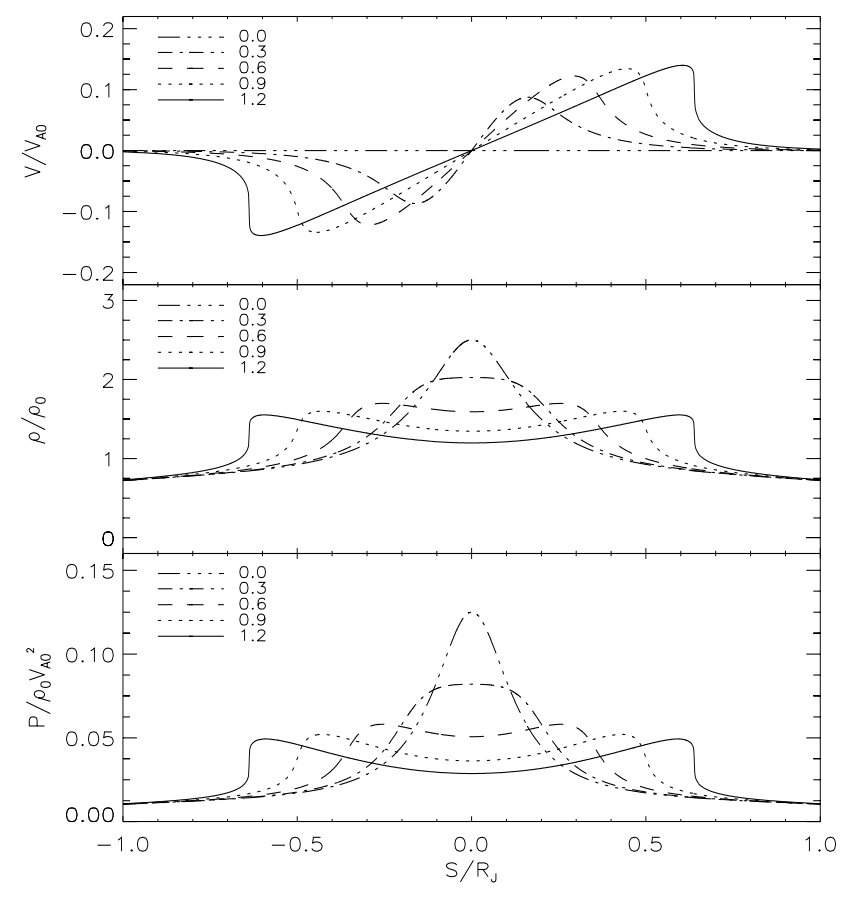

Fig. 2. The initial stage of the slow mode generation. From top to bottom, the distributions of the normalized velocity, plasma density and pressure are shown for different times in units $R_{J} / V_{A 0}$. This case corresponds to the parameters $\kappa=2, P_{1} / P_{0}=6, \beta_{0}=0.04$.

formation of the slow shock, produced by the local enhancement of the plasma pressure at the initial moment. After that, when the shock front is formed, we calculate the propagation of the slow shock along the magnetic flux tube towards the planet by separating the shock front and using system (16-18) with the adiabatic Eq. (19) behind the shock. This separation of the shock front allows us to obtain more accurate results. When a nonlinear MHD slow wave is evolved into a shock, the shock front must be separated with the jump conditions for a slow shock.

To solve the problem, we use the conservative, two-step finite difference numerical scheme of Godunov (Godunov and Ryabenkii, 1987) with a constant grid size for $\alpha, \alpha_{i}=i \Delta \alpha$. At the first step, the method of characteristics is used to calculate the density, velocity, and magnetic field at intermediate grid points labeled by half-integer numbers. On the second step, the plasma parameters and the magnetic field are calculated at the main grid points labeled with integer numbers

$$
\begin{gathered}
V_{i}^{n+1}=V_{i}^{n}-\frac{1}{B_{i}} \frac{\Delta \tau}{\Delta \alpha}\left[P_{i+1 / 2}^{n+1 / 2}-P_{i-1 / 2}^{n+1 / 2}\right]+\Delta \tau F_{i}^{n}, \\
\left(\frac{B}{\rho}\right)_{i}^{n+1}=\left(\frac{B}{\rho}\right)_{i}^{n}+\frac{\Delta \tau}{\Delta \alpha}\left[V_{i+1 / 2}^{n+1 / 2}-V_{i-1 / 2}^{n+1 / 2}\right] .
\end{gathered}
$$

Each time step is determined from the Courant-FriedrichsLewy (CFL) condition which provides the stability of the numerical scheme. Here, the CFL condition is determined by

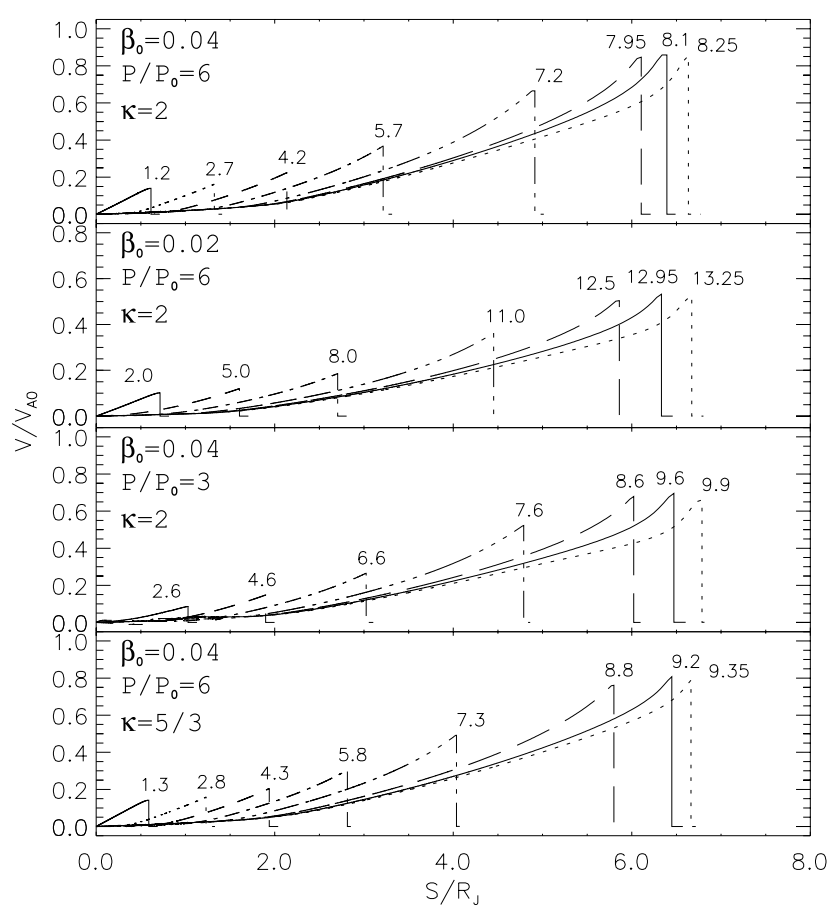

Fig. 3. Propagation of the slow shock along the flux tube. Distribution of the plasma velocity are shown as functions of the distance along the magnetic tube for different times.

the characteristics for our equations in material coordinates $\max \left[\left(C_{s} \rho / B\right)(\Delta \tau / \Delta \alpha)\right] \leq 1$,

where $C_{s}$ is a local sonic speed $\left(C_{s}=\sqrt{\kappa P / \rho}\right), \Delta \alpha$ is the grid size for the $\alpha$ coordinate, and $\Delta \tau$ is the time step. For $\Delta \alpha=5.10^{-5}$, the time step is $\Delta \tau=8.4810^{-5}$ at the initial moment, and it is equal to $\Delta \tau=3.5210^{-4}$ at the final moment.

The numerical procedure is stable, and we checked the convergence of our numerical results by taking different grid sizes. Changing the grid size by a factor of $2, \Delta \alpha=2.510^{-5}$ and $\Delta \alpha=5 \cdot 10^{-5}$, we obtained a very small difference in the numerical solutions. For these two steps, the velocity maxima are $V_{\max }=0.8603$ and $V_{\max }=0.859$, respectively.

\section{Results of MHD simulations}

Figure 2 shows the first stage of the slow-mode generation. The initial pressure pulse decays into two nonlinear slow waves propagating along the flux tube in opposite directions. The amplitudes of these waves are decreasing in the course of time, the leading fronts are becoming more and more steep, and eventually, the slow waves are converted into shocks. From top to bottom, the distributions of the normalized velocity, density, and plasma pressure are shown for different times in units $R_{J} / V_{A 0}$.

If the cross section of the magnetic flux tube would no longer change, the process of wave propagation could be easily predicted: slow shocks would travel along the flux tube, 


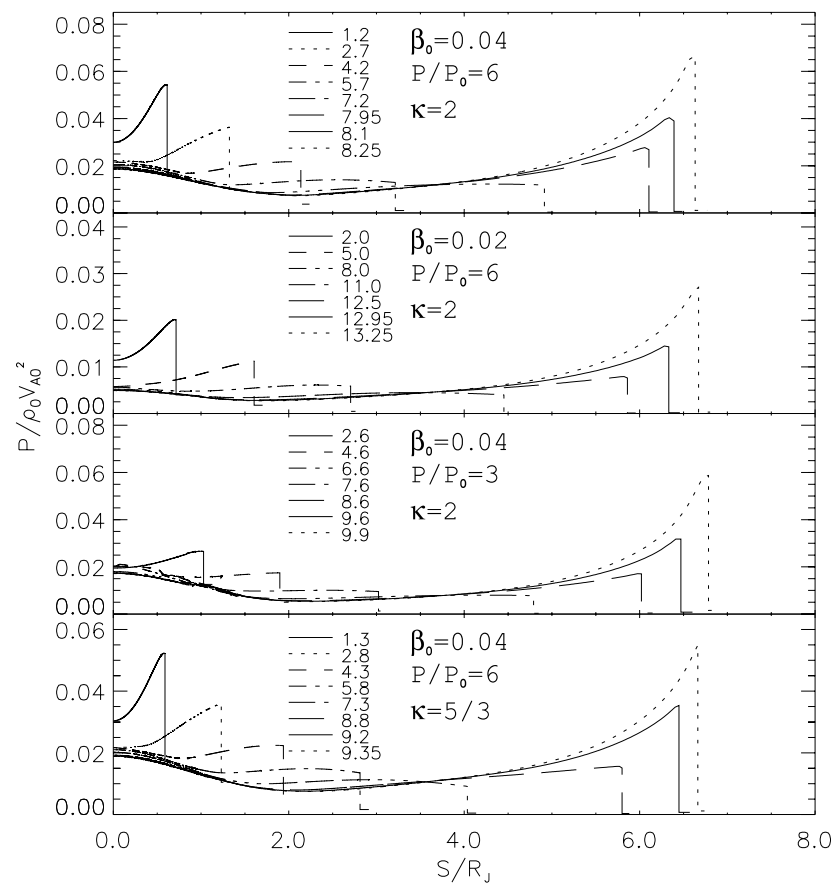

Fig. 4. Distributions of the plasma pressure as functions of the distance along the tube for different times.

thereby being gradually damped with decreasing flow velocity behind the shock front.

However, the flux tube cross section is inversely proportional to the magnetic field strength and therefore, it has to decrease as $r^{3}$ decreases due to the dipole field configuration. Hence, the plasma flow has to move into a flux tube that becomes narrower and narrower.

Figures 3, 4, and 5 show distributions of the plasma velocity, pressure and density as functions of the distance along the magnetic flux tube for different cases. From top to bottom, we use

$\beta_{0}=0.04, \quad P_{1} / P_{0}=6, \quad \kappa=2 ;$

$\beta_{0}=0.02, \quad P_{1} / P_{0}=6, \quad \kappa=2$;

$\beta_{0}=0.04, \quad P_{1} / P_{0}=3, \quad \kappa=2$;

$\beta_{0}=0.04, \quad P_{1} / P_{0}=6, \quad \kappa=5 / 3$.

Here, $P_{1}$ is the amplitude of the initial pressure pulse.

The different shock positions correspond to the different times scaled to $R_{J} / V_{A 0}$. Comparing the first and the second panel (from top to bottom), one can see the effect of the variation of the initial background beta parameters $\left(\beta_{0}=0.04\right.$, and $\beta_{0}=0.02$ ) for a fixed amplitude of the pressure pulse $\left(P_{1} / P_{0}=6\right)$. An increase in the initial plasma beta brings about an enhancement of the velocity maximum with respect to the initial Alfvén speed.

Comparing the first and the third panels, one can see the effect of the variation in the pressure pulse amplitude. An increase in the pressure pulse amplitude leads to an enhancement of the velocity maximum. The ratio of the velocity

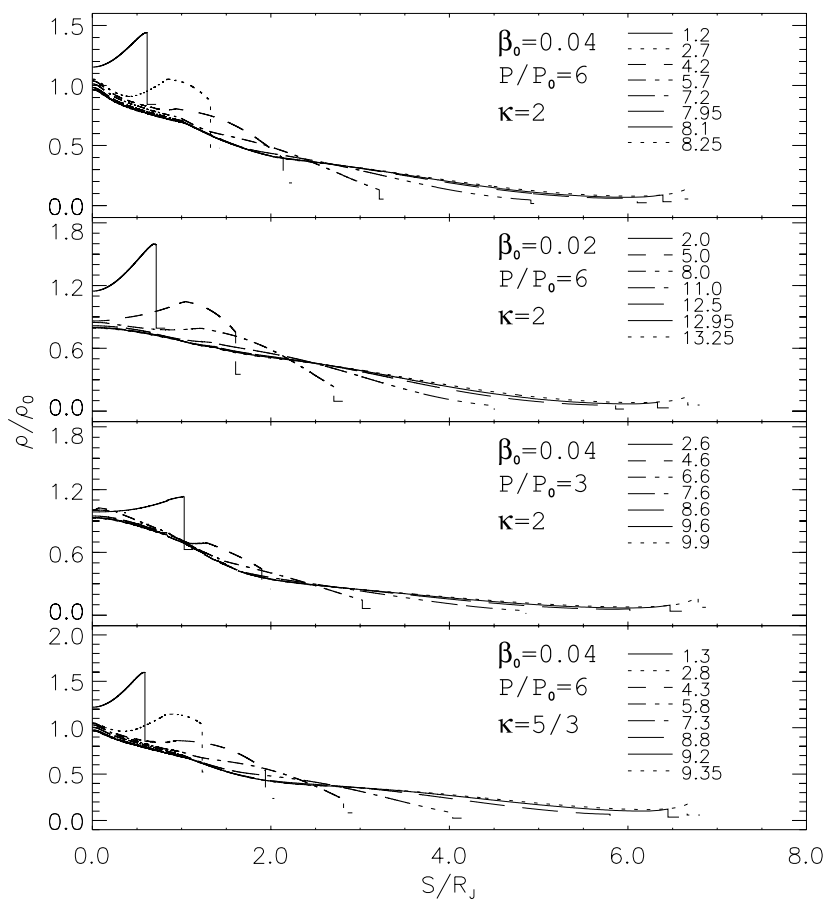

Fig. 5. Distributions of the plasma density as functions of the distance along the tube for different times.

maxima is a bit less than the square root of the ratio of the pressure pulse amplitudes.

Comparing panels 1 and 4, one can see that the variation of the polytropic exponent has no great influence on the results of the calculations.

The different behaviours of plasma pressure and density as shown in Figs. 4 and 5, can be explained as follows: a decrease in the density calculated just behind the shock is related to the fall in the background density caused by the centrifugal force. However, an increase in the shock intensity plays no crucial rule, because the density jump at the shock is limited by a constant value depending on the polytropic exponent.

At the initial stage of the wave propagation, the plasma pressure decreases for the same reason as for the density (decrease in the background pressure). However, for a sufficiently large $t$, the pressure has a well pronounced enhancement, due to the pressure jump at the shock being much larger than the density jump. Concerning the background parameters, plasma pressure and density are proportional to each other because the undisturbed temperature is assumed to be constant.

Figure 6 represents the quantities calculated just after the shock front as functions of the distance traveled by the shock. From top to bottom, the first three panels of Fig. 6 show velocity, density, and plasma pressure just behind the shock as functions of the travel distance of the shock for different input parameters. The lowest curve corresponds to the smallest initial beta. The bottom panel shows the distance to the dipole center and the travel time of the shock as functions of 


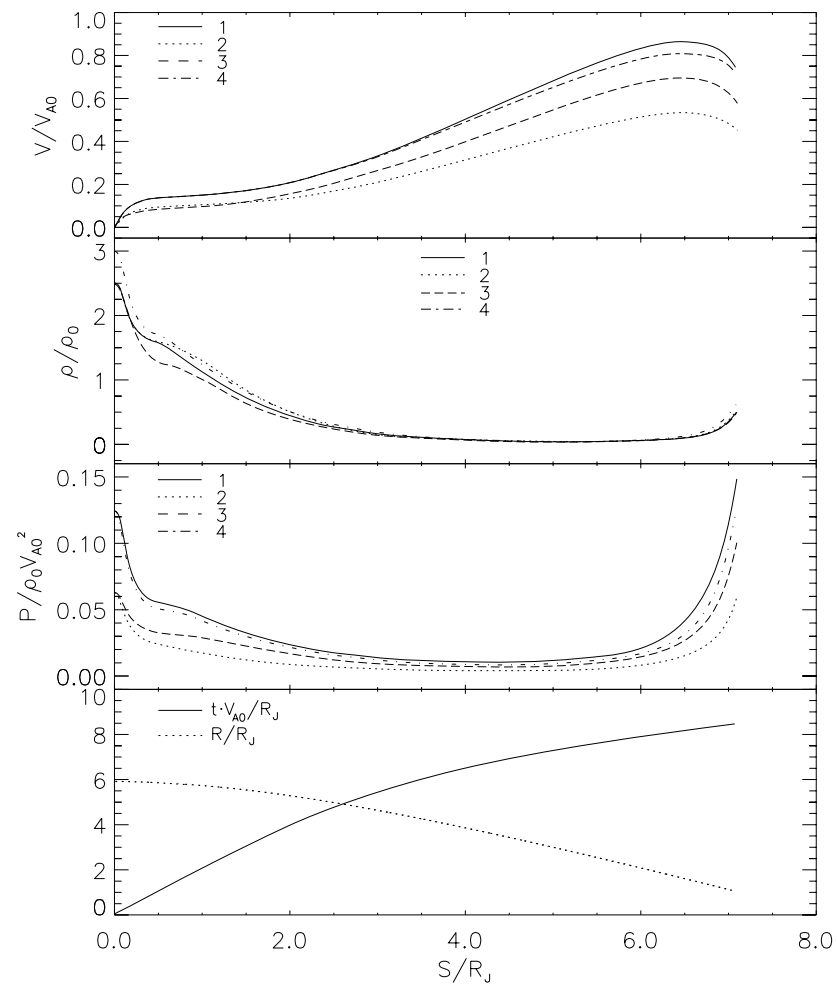

Fig. 6. The first three panels correspond to velocity, density and plasma pressure just behind the shock as functions of the travel distance of the shock for the different input parameters: (1) $\beta_{0}=$ $0.04, P_{1} / P_{0}=6, \kappa=2$; (2) $\beta_{0}=0.02, P_{1} / P_{0}=6, \kappa=2$; (3) $\beta_{0}=0.04, P_{1} / P_{0}=3, \kappa=2$; (4) $\beta_{0}=0.04, P_{1} / P_{0}=6, \kappa=$ $5 / 3$. Bottom panel: Distance to the dipole center and travel time of the shock as functions of the travel distance.

the travel distance.

This figure indicates clearly the different stages of the wave propagation. At the first stage $\left(S<0.5 R_{J}\right)$, the nonlinear MHD slow wave produced by the pressure pulse is evolved into the shock. At the second stage $\left(0.5 R_{J}<S<\right.$ $2 R_{J}$ ), the wave amplitude characterized by the plasma velocity behind the shock is nearly constant. At this stage, a shock damping is compensated by two factors: the decrease in the background density due to the centrifugal effect, and the gradual decrease in the magnetic tube cross section. During this period, the plasma pressure behind the shock decreases due to the fall into the background pressure caused by the centrifugal force. At the third stage $\left(S>2 R_{J}\right)$, the wave amplitude starts to enhance due to a rapid increase in the magnetic field strength and a corresponding decrease in the magnetic tube cross section. The velocity reaches its maximum value by the arrival at the point $\left(S \sim 6.4 \pm 0.1 R_{J}\right)$ for all cases. After this maximum point, the velocity starts to decrease as a result of the enhancement of the background pressure due to the gravitational force.

Figure 7 shows the comparison of velocities calculated just behind the shock for rotating and nonrotating magnetic flux tubes. One can see that the velocity enhancement is much more pronounced in the case of a rotating magnetic tube, in

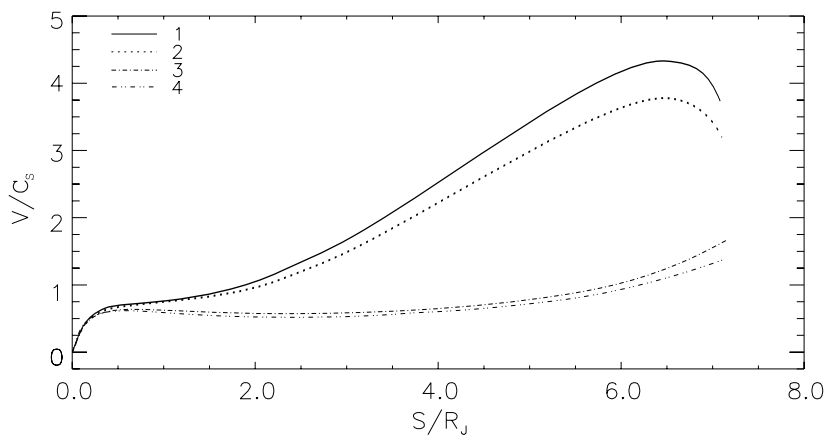

Fig. 7. Comparison of the velocities just behind the shock calculated for rotating $(1,2)$ and nonrotating $(3,4)$ tubes for the different initial betas. Curves 1,3 correspond to $\beta_{0}=0.04$, and curves 2,4 correspond to $\beta=0.02$. The velocity is normalized to the background sonic speed $C_{S}$.

which the centrifugal potential affects substantially the distribution of the background plasma pressure and density along the tube.

As it was shown, the slow wave propagation in a flux tube with an increasing magnetic field and correspondingly decreasing tube cross section is considerably different from that for a homogeneous magnetic field. The narrow channel effect leads to an intensification of the propagating wave rather than to damping due to its expansion. This effect might be important for the Sun or other planets where pressure pulses can be produced inside thin flux tubes.

\section{Discussion and conclusions}

In the case of converging magnetic field lines, such as in a dipole magnetic field, the propagation of MHD slow shocks is studied numerically using the ideal MHD equations in an approximation suitable for a thin magnetic flux tube with low plasma beta. The result obtained in the numerical study shows that the intensity of the MHD slow shock increases greatly while the shock is propagating along the narrowing magnetic flux tube.

This effect has a simple physical explanation in the case of linear wave perturbations. Let us consider a pressure pulse of duration $\Delta t$. For simplicity, we consider a linear magnetosonic wave perturbations generated by the pulse. This pressure pulse produces a wave packet of duration $\Delta t$ propagating along the magnetic flux tube. The energy of this wave perturbation can be estimated as

$W=\frac{1}{2} \Sigma \rho_{0} v^{2} C_{s} \Delta t$.

Here, $\Sigma$ is the cross section of the magnetic flux tube.

Taking into account the behaviour of the cross section along the tube, e.g.

$\Sigma=\frac{\Sigma_{0} B_{0}}{B}$, 
we find a relation between the wave amplitude and the magnetic field strength

$v=\sqrt{\frac{2 B W}{\Sigma_{0} B_{0} \rho_{0} C_{s} \Delta t}}$.

The wave energy is proportional to the amplitude of the pressure pulse, and thus the wave amplitude is proportional to the square root of the pressure pulse. One can also see that in the narrowing magnetic flux tube, a linear wave amplitude should increase proportionally to the square root of the magnetic field strength. However, in the nonlinear case, this effect is less pronounced due to plasma heating at the shock front.

The centrifugal force caused by the rotation of the magnetic flux tube plays an important role in the process of slow wave evolution. In the rotating magnetic tube, the background density and plasma pressure increase along the tube towards the equator. In such a case, a slow wave propagating from the equator with decreasing density and plasma pressure is evolving into the shock much faster than that in the case of a nonrotating tube with constant background parameters.

The model is applied to the case of the interaction between Jupiter and Io. The latter is considered as a source of plasma pressure pulses. One aspect of the Io-Jupiter interaction is that of Alfvén waves, generated by Io moving in a magnetized plasma (Bagenal, 1983; Menietti and Curran, 1990). Our study focuses on an additional mechanism of the interaction between Io and Jupiter based on MHD slow shocks propagating from Io towards Jupiter along a magnetic flux tube. We believe that the slow mode waves are not only important in the course of the torus plasma flow around Io (Kopp, 1996; Linker et al., 1991), but they can also be responsible for specific phenomena, such as aurora or DAM radiation, together with Alfvén waves.

As far as the aurora is concerned, we suggest the following interpretation of the slow wave input which is considered to be complementary to the Alfvén mechanism. Direct observations of the Io footprint aurora show that there is a bright leading point corresponding to the projection of Io. In addition, a diffuse fainter emission is observed, which is extended in longitude with several bright spots in the tail (Connerney et al., 1999). The leader is certainly connected with the first Alfvén wave arrival at the Jovian ionosphere. The trailing spots have been interpreted as arrivals of the reflected Alfvén waves (Connerney et al., 1999). It is our point that one of these bright spots in the tail might be connected with the arrival of a slow shock.

With regard to DAM observations, Genova and Aubier (1985) found the lag in the range of $50^{\circ}-80^{\circ}$ between the instantaneous position of the Io flux tube and the source region of decametric radio emissions. The observational estimations of the lag were diminished in recent studies by Queinnec and Zarka (1998). They showed that some parts of the DAM emission have $30^{\circ}-50^{\circ}$ lag. The lag calculated in our model for the MHD slow shocks is about $45^{\circ}$.
Acknowledgements. This work is supported by the INTAS-ESA project 99-01277. It is also supported in part by grants No. 01-05-65070 and No. 01-05-64954 from the Russian Foundation of Basic Research. Part of this work is supported by the "Fonds zur Förderung der wissenschaftlichen Forschung", under projects P12761-TPH and P14744-TPH. This work is further supported by grant No 01-05-02003 from the Russian Foundation of Basic Research and by project I.4/2001 from "Österreichischer Akademischer Austauschdienst". We acknowledge support by the Austrian Academy of Sciences, "Verwaltungsstelle für Auslandsbeziehungen".

\section{Appendix A Introduction of a mass variable and Euler potentials as material coordinates}

In this subsection, we prove that the mass coordinate and Euler potentials used in our solution are constant along trajectories of fluid particles, and thus they are material coordinates.

We introduce the Euler potentials, $\phi$ and $\psi$, determined by the equation

$\boldsymbol{B}=\nabla \phi \times \nabla \psi$.

These potentials are constant along a magnetic field line and satisfy the equations

$\boldsymbol{B} \cdot \nabla \phi=0, \quad \boldsymbol{B} \cdot \nabla \psi=0$.

Using these equations, we can determine the potentials for all magnetic field lines. Near the planet, these potentials $\phi$ and $\psi$ can be considered to be equal to the magnetic latitude and longitude, respectively.

For each magnetic field line characterized by two constant parameters $\phi$ and $\psi$, we define a function $\alpha(S, \phi, \psi)$ depending on the distance $S$ along the magnetic field line as follows

$\alpha=\int_{0}^{S} \rho / B d S^{\prime}$.

From this definition it follows that

$\boldsymbol{B} \cdot \nabla \alpha=\rho$.

In our definition, the quantity $\alpha$ and distance $S$ are equal to zero at the equator.

Finally, we have three quantities $\alpha, \phi$, and $\psi$ which can be used as independent coordinates. Now we have to prove that these quantities are material coordinates.

We consider Eqs. (A2, A4) as a linear algebraic system with respect to the three unknown quantities $B_{x}, B_{y}, B_{z}$. A solution of this algebraic system is

$$
\begin{gathered}
B_{x}=\frac{\rho}{J} \frac{D(\phi, \psi)}{D(y, z)}, \quad B_{y}=\frac{1}{J} \frac{D(\phi, \psi)}{D(z, x)}, \\
B_{z}=\frac{1}{J} \frac{D(\phi, \psi)}{D(x, y)},
\end{gathered}
$$

where $J$ is a Jacobian

$J=\frac{D(\alpha, \phi, \psi)}{D(x, y, z)}$. 
In vector form, Eqs. (A5) can be written as follows

$$
\boldsymbol{B}=\frac{\rho}{J} \nabla \phi \times \nabla \psi
$$

Comparing (A7) and (A1), we find the Jacobian $J=\rho$. Using the standard technique of Jacobian transformations, we find that

$$
\begin{aligned}
B_{x} & =\frac{\rho}{J} \frac{D(\phi, \psi)}{D(y, z)}=\frac{\rho}{J} \frac{D(x, \phi, \psi)}{D(x, y, z)} \\
& =\rho \frac{D(x, y, z)}{D(\alpha, \phi, \psi)} \frac{D(x, \phi, \psi)}{D(x, y, z)} \\
& =\rho \frac{D(x, \phi, \psi)}{D(\alpha, \phi, \psi)}=\rho \frac{\partial x}{\partial \alpha} .
\end{aligned}
$$

Using similar transformations for the other components, we obtain

$B_{y}=\rho \frac{\partial y}{\partial \alpha}, \quad B_{z}=\rho \frac{\partial z}{\partial \alpha}$.

The last three equations can be written in a vector form

$\boldsymbol{B}=\rho \frac{\partial \boldsymbol{r}}{\partial \alpha}$.

Furthermore, we use the induction equation for the frozenin magnetic field, which can be written in the form

$\frac{d}{d t}\left(\frac{\boldsymbol{B}}{\rho}\right)=\left(\frac{\boldsymbol{B}}{\rho} \cdot \nabla\right) \boldsymbol{V}$.

Here $d() / d t$ is the derivative along the trajectory of a fluid particle defined as

$$
\begin{aligned}
& \frac{d()}{d t}=\frac{\partial()}{\partial t}_{x, y, z}+\boldsymbol{V} \cdot \nabla()= \\
& \frac{\partial()}{\partial t}_{\alpha, \phi, \psi}+\frac{d \alpha}{d t} \frac{\partial()}{\partial \alpha}+\frac{d \phi}{d t} \frac{\partial()}{\partial \phi}+\frac{d \psi}{d t} \frac{\partial()}{\partial \psi} .
\end{aligned}
$$

Using (A10), (A2), (A4) and (A11), and considering $\boldsymbol{B}$ and $\rho$ to be functions of $(\alpha, \phi, \psi, t)$, we get the equation

$\frac{d}{d t}\left(\frac{\partial \boldsymbol{r}}{\partial \alpha}\right)=\frac{\partial \boldsymbol{V}}{\partial \alpha}$.

Taking into account a kinematic relation between the velocity and radius vector as given in relation (A12), we get

$$
\boldsymbol{V}=\frac{d \boldsymbol{r}}{d t}=\frac{\partial \boldsymbol{r}}{\partial t}_{\alpha, \phi, \psi}+\frac{d \alpha}{d t} \frac{\partial \boldsymbol{r}}{\partial \alpha}+\frac{d \phi}{d t} \frac{\partial \boldsymbol{r}}{\partial \phi}+\frac{d \psi}{d t} \frac{\partial \boldsymbol{r}}{\partial \psi}
$$

Substituting (A10) and (A14) into Eq. (A13) and using (A12), we finally obtain

$$
\begin{aligned}
\frac{\partial^{2} \boldsymbol{r}}{\partial \alpha \partial t}+\frac{d \alpha}{d t} \frac{\partial}{\partial \alpha}\left(\frac{\partial \boldsymbol{r}}{\partial \alpha}\right) & \\
+ & \frac{d \phi}{d t} \frac{\partial}{\partial \phi}\left(\frac{\partial \boldsymbol{r}}{\partial \alpha}\right)+\frac{d \psi}{d t} \frac{\partial}{\partial \psi}\left(\frac{\partial \boldsymbol{r}}{\partial \alpha}\right) \\
= & \frac{\partial^{2} \boldsymbol{r}}{\partial t \partial \alpha}+\frac{\partial}{\partial \alpha}\left(\frac{d \alpha}{d t} \frac{\partial \boldsymbol{r}}{\partial \alpha}\right) \\
+ & \frac{\partial}{\partial \alpha}\left(\frac{d \phi}{d t} \frac{\partial \boldsymbol{r}}{\partial \phi}\right)+\frac{\partial}{\partial \alpha}\left(\frac{d \psi}{d t} \frac{\partial \boldsymbol{r}}{\partial \psi}\right) .
\end{aligned}
$$

The last equation can be simplified to

$$
\frac{\partial \boldsymbol{r}}{\partial \alpha} \frac{\partial \dot{\alpha}}{\partial \alpha}+\frac{\partial \boldsymbol{r}}{\partial \phi} \frac{\partial \dot{\phi}}{\partial \alpha}+\frac{\partial \boldsymbol{r}}{\partial \psi} \frac{\partial \dot{\psi}}{\partial \alpha}=0 .
$$

Here

$\dot{\alpha}=\frac{d \alpha}{d t}, \quad \dot{\phi}=\frac{d \phi}{d t}, \quad \dot{\psi}=\frac{d \psi}{d t}$.

We consider the vector Eq. (A16) as three scalar homogeneous algebraic equations with regard to the quantities $\partial(\dot{\alpha}) / \partial \alpha, \partial(\dot{\phi}) / \partial \alpha, \partial(\dot{\psi}) / \partial \alpha$.

The determinant of this system is the Jacobian $J$ that is not equal zero (see above, $J=\rho$ ). For a nonzero determinant, this system has only the trivial solution if

$\frac{\partial \dot{\alpha}}{\partial \alpha}=0, \quad \frac{\partial \dot{\phi}}{\partial \alpha}=0, \quad \frac{\partial \dot{\psi}}{\partial \alpha}=0$.

According to our definition, for each magnetic field line, quantity $\alpha$ is equal to zero at the equator. This condition and Eq. (A17) bring about $\dot{\alpha}=0$ everywhere.

The Euler potentials are equal to latitude and longitude of the magnetic field line near the planet, where $\dot{\phi}=0$ and $\dot{\psi}=$ 0 . These boundary conditions for Euler potentials together with equations (A17) lead to the equations $\dot{\phi}=0$ and $\dot{\psi}=0$ everywhere.

The proved statement is

$\frac{d \alpha}{d t}=0, \quad \frac{d \phi}{d t}=0, \quad \frac{d \psi}{d t}=0$.

Therefore, the introduced independent variables $\alpha, \phi, \psi$ are material coordinates.

From Eqs. (A14) and (A18) we obtain a simple relation between velocity and radius vector:

$\boldsymbol{V}=\frac{\partial \boldsymbol{r}}{\partial t}_{\alpha, \phi, \psi}$.

Using Eqs. (A2), (A4) and (A18), the dimensionless momentum equation can be transformed as follows

$$
\begin{aligned}
\frac{d \boldsymbol{V}}{d t} & +\frac{1}{\rho} \nabla \Pi-\frac{1}{\rho}(\boldsymbol{B} \cdot \nabla) \boldsymbol{B}-\boldsymbol{F} \\
& =\frac{\partial \boldsymbol{V}}{\partial t}+\frac{1}{\rho} \nabla \Pi-\frac{1}{\rho} \frac{\partial \boldsymbol{B}}{\partial \alpha}(\boldsymbol{B} \cdot \nabla \alpha) \\
& -\frac{1}{\rho} \frac{\partial \boldsymbol{B}}{\partial \phi}(\boldsymbol{B} \cdot \nabla \phi)-\frac{1}{\rho} \frac{\partial \boldsymbol{B}}{\partial \psi}(\boldsymbol{B} \cdot \nabla \psi)-\boldsymbol{F} \\
& =\frac{\partial \boldsymbol{V}}{\partial t}+\frac{1}{\rho} \nabla \Pi-\frac{\partial \boldsymbol{B}}{\partial \alpha}-\boldsymbol{F}=0 .
\end{aligned}
$$

Using Eqs. (A2), (A4), and (A18), we transform the induction Eq. (A11)

$$
\begin{aligned}
\frac{d}{d t}\left(\frac{\boldsymbol{B}}{\rho}\right)-\left(\frac{\boldsymbol{B}}{\rho} \cdot \nabla\right) \boldsymbol{V} \\
\quad=\frac{\partial}{\partial t}\left(\frac{\boldsymbol{B}}{\rho}\right)-\frac{\partial \boldsymbol{V}}{\partial \alpha}=0 .
\end{aligned}
$$

The MHD system is closed by the adiabatic equation

$\frac{d}{d t}\left(P / \rho^{\kappa}\right)=\frac{\partial}{\partial t}\left(P / \rho^{\kappa}\right)=0$. 


\section{Appendix B Energy equation}

Equation (A20) multiplied by the vector $\boldsymbol{V}$ yields a scalar equation

$\frac{\partial V^{2} / 2}{\partial t}-\boldsymbol{V} \cdot \frac{\partial \boldsymbol{B}}{\partial \alpha}+\frac{1}{\rho} \nabla \Pi \cdot \boldsymbol{V}=\boldsymbol{F} \cdot \boldsymbol{V}$.

Using equations

$$
\begin{gathered}
\boldsymbol{V}=\frac{\partial \boldsymbol{r}}{\partial t}, \quad \frac{\partial \boldsymbol{V}}{\partial \alpha}=\frac{\partial}{\partial t}\left(\frac{\boldsymbol{B}}{\rho}\right), \\
P=\Pi-B^{2} / 2, \quad \frac{\partial}{\partial t}\left(\frac{P}{\rho^{\kappa}}\right)=0,
\end{gathered}
$$

and assuming that

$\Pi=\Pi(x, y, z)$,

and using the relations

$\frac{\partial \Pi}{\partial t}=\nabla \Pi \cdot \frac{\partial \boldsymbol{r}}{\partial t}, \quad \boldsymbol{F}=-\nabla \Phi$,

we transform Eq. (B1) as follows

$$
\begin{aligned}
& \frac{\partial V^{2} / 2}{\partial t}-\boldsymbol{V} \cdot \frac{\partial \boldsymbol{B}}{\partial \alpha}+\frac{1}{\rho} \nabla \Pi \cdot \frac{\partial \boldsymbol{r}}{\partial t}+\nabla \Phi \cdot \frac{\partial \boldsymbol{r}}{\partial t} \\
& =\frac{\partial V^{2} / 2}{\partial t}-\frac{\partial(\boldsymbol{B} \cdot \boldsymbol{V})}{\partial \alpha}+\boldsymbol{B} \cdot \frac{\partial}{\partial t}\left(\frac{\boldsymbol{B}}{\rho}\right)+\frac{1}{\rho} \frac{\partial \Pi}{\partial t}+\frac{\partial \Phi}{\partial t} \\
& \quad=\frac{\partial}{\partial t}\left(\frac{V^{2}}{2}+\frac{B^{2}}{2 \rho}+\frac{\Pi}{\rho}+\Phi\right)-\frac{\partial(\boldsymbol{B} \cdot \boldsymbol{V})}{\partial \alpha} \\
& \quad+\left(\Pi-\frac{B^{2}}{2}\right) \frac{1}{\rho^{2}} \frac{\partial \rho}{\partial t} \\
& =\frac{\partial}{\partial t}\left(\frac{V^{2}}{2}+\frac{B^{2}}{2 \rho}+\frac{P}{(\kappa-1) \rho}+\frac{\Pi}{\rho}+\Phi\right) \\
& -\frac{\partial(\boldsymbol{B} \cdot \boldsymbol{V})}{\partial \alpha}=0 .
\end{aligned}
$$

\section{Appendix C Estimation of the Alfvén perturbations produced by a pressure pulse}

For an Alfvén wave, perturbations of velocity and magnetic field are proportional to each other

$v_{\perp}=\frac{1}{\sqrt{\mu_{0} \rho_{0}}} b_{\perp}$.

We consider a local deformation of the magnetic flux tube caused by an enhancement of the plasma pressure. The enhanced plasma pressure thickens the cross section of the tube. This local expansion of the magnetic flux tube can generate a slow magnetosonic wave, as well as an Alfvén wave.

For simplicity, we consider the cross section of the tube as a circle. We introduce perpendicular and parallel components of the disturbed magnetic field, which are defined with respect to the center line of the tube. A deformation of the magnetic flux tube is determined by a function $\delta=\delta(S)$, where $\delta$ is the perturbation of the radius of the tube.

The perturbation of the magnetic tube radius is related to the perpendicular magnetic field component $B_{\perp}$ as follows

$b_{\perp}=B_{\|} \frac{d \delta}{d S} \sim B_{0} \frac{\delta}{\lambda}$,

where subscript 0 denotes undisturbed parameters, and $\lambda$ is the length scale of the perturbation.

The conservation law for the magnetic flux in the tube yields

$\left(B_{0}+b_{\|}\right)(\Delta+\delta)^{2}=B \Delta^{2}$,

where $\Delta$ is the undisturbed radius of the given magnetic flux tube. For linear perturbations, the last equation is simplified to

$b_{\|}=-2 B_{0} \frac{\delta}{\Delta}$.

From Eqs. (C2) and (C4), we find a relation between $b_{\perp}$ and $b_{\|}$

$b_{\perp}=-\frac{1}{2} \frac{\Delta}{\lambda} b_{\|}$.

For a given total pressure, the parallel perturbation of the magnetic field can be estimated as

$b_{\|}=-\mu_{0}\left(P-P_{0}\right) / B \sim \frac{\eta-1}{2} B_{0} \beta_{0}$.

Here, $\eta=P_{1} / P_{0}$ is the ratio of the initial pressure maximum to the background pressure at the equator. In our model, this ratio is taken to be 6 or less.

Using the equations above, we obtain

$v_{\perp} \sim \frac{\Delta}{\lambda} \frac{\eta-1}{4} \frac{B_{0}}{\sqrt{\mu_{0} \rho}} \beta_{0}$.

The energy of an Alfvén wave can be estimated as

$W_{a} \sim \frac{1}{2} \rho v_{\perp}^{2} \Sigma \lambda \sim \frac{(\eta-1)^{2}}{32} \rho V_{A}^{2} \frac{\Delta^{2}}{\lambda} \beta_{0}^{2} \Sigma$.

The energy of a slow wave can be estimated as

$W_{s} \sim \frac{1}{\kappa-1} P \lambda \Sigma$,

where $\kappa$ is the polytropic exponent.

Taking the ratio of the energies as estimated above, we find

$W_{A} / W_{s} \sim \frac{(\eta-1)^{2}}{32} \rho \frac{V_{A}^{2}}{P} \frac{\Delta^{2}}{\lambda^{2}} \beta_{0}^{2} \leq \frac{(\eta-1)^{2}}{16 \eta} \frac{\Delta^{2}}{\lambda^{2}} \beta_{0}$.

Taking $\Delta \sim \lambda$, and $\eta \sim 6$, we obtain $W_{A} / W_{s} \sim 0.3 \beta_{0}$.

Thus, the Alfvén wave energy produced by a pressure pulse is much less than that of the slow magnetosonic wave. 


\section{Appendix D Estimation of the plasma velocity behind the slow shock front}

The plasma velocity and the plasma pressure behind the shock propagating along the magnetic field are

$V=D\left(1-\rho^{*} / \rho\right)$

$P=\rho^{*} D^{2}\left(1-\rho^{*} / \rho\right)+P^{*}$.

Here, $D$ is the shock speed, the parameters denoted by the symbol $*$ correspond to the background conditions before the shock.

From the equations above, we obtain a relation between the velocity and plasma pressure

$V=\left(P-P^{*}\right)^{1 / 2}\left(\frac{\rho-\rho^{*}}{\rho \rho^{*}}\right)^{1 / 2}$.

The latter can be written in dimensionless form

$$
\begin{aligned}
\tilde{V} & =\frac{V}{V_{A 0}} \\
& =\sqrt{\frac{P_{0}}{\rho_{0} V_{A 0}^{2}}} \sqrt{\frac{\left(P-P^{*}\right)}{P_{0}}} \sqrt{\frac{\rho_{0}}{\rho^{*}}-\frac{\rho_{0}}{\rho}} \propto \sqrt{\beta_{0}} .
\end{aligned}
$$

The shock speed is

$$
\begin{aligned}
\tilde{D} & =\frac{D}{V_{A 0}}=\sqrt{\frac{\left(P-P^{*}\right)}{V_{A 0}^{2}}} \sqrt{\frac{\rho}{\rho^{*}\left(\rho-\rho^{*}\right)}} \\
& =\sqrt{\beta_{0}} \sqrt{\frac{P-P^{*}}{2 P_{0}}} \sqrt{\frac{\rho \rho_{0}}{\rho^{*}\left(\rho-\rho^{*}\right)}} \propto \sqrt{\beta_{0}} .
\end{aligned}
$$

Here, the pressure $P$ is smaller than the amplitude of the initial pressure pulse $P_{1}$, the background pressure $P^{*}$ is much smaller than $P$, and thus the ratio of the pressures is estimated as

$$
\frac{P-P^{*}}{P_{0}}<\frac{P_{1}}{P_{0}}
$$

The ratios of the densities $\rho / \rho_{0}$ and $\rho / \rho^{*}$ are also limited.

Hence, the normalized velocity and shock speed are

$$
V / V_{A 0}=\sqrt{\beta_{0}} V^{(0)}, \quad D / V_{A 0}=\sqrt{\beta_{0}} D^{(0)} .
$$

The dimensionless time of the wave propagation along the tube can be estimated as

$\tilde{t}=\frac{L^{*} V_{A 0}}{D R_{J}}=\frac{L^{*}}{R_{J}} \frac{1}{\sqrt{\beta_{0}} D^{(0)}} \propto \frac{1}{\sqrt{\beta_{0}}}$,

where $L^{*}$ is the length of the magnetic tube.

\section{References}

Bagenal, F.: Alfvén wave propagation in the Io plasma torus, J. Geophys. Res., 88, 3013-3025, 1983.

Bagenal, F.: Empirical model of the Io plasma torus: Voyager measurements, J. Geophys. Res., 99, 11 043-11 062, 1994.

Connerney, J. E. P., Sato, T., and Clarke, J. T.: Tracking the Io flux tube footpoints, report presented at "The 31st Annual Meeting of the AAS Division for Planetary Sciences", 10-15 October 1999, Padova, Italy, 1185, 1999.

Combi, M. R., Kabin, K., Gombosi, T. I., DeZeeuw, D. L., and Powell, K. G.: Io's plasma environment during the Galileo flyby: Global three-dimensional modeling with adaptive mesh refinement, J. Geophys. Res., 103, A5, 9071-9081, 1998.

Chen, C. X. and Wolf, R. A.: Theory of thin-filament motion in Earth's magnetotail and its application to bursty bulk flow, J. Geophys. Res., 104, A7, 14 613-14 626, 1999.

Erkaev, N. V. and Mezentsev, A. V.: The flow of the solar wind around the magnetosphere and the generation of electric fields, in: Solar Wind - Magnetosphere Interaction, (Eds) Heyn, M. F., Biernat, H. K., Semenov, V. S., and Rijnbeek, R. P., (Österreichische Akademie der Wissenschaften, Vienna), 43-66, 1992.

Erkaev, N. V., Farrugia, C. J., and Biernat, H. K.: Effects on the Jovian magnetosheath arising from solar wind flow around nonaxial bodies, J. Geophys. Res., 101, 10 665-10 672, 1996.

Fisher, G. H., Fan, Y., Linton, M. G., and Abbett, W. P.: Magnetic flux tubes inside the Sun, Physics of Plasmas, 7, 5, 2173-2179, 2000.

Frank, L. A., Paterson, W. R., Ackerson, K. L., Vasyliunas, V. M., Coronity, F. V., and Bolton, S. J.: Plasma observations at Io with the Galileo spacecraft, Science, 274, 394-395, 1996.

Genova, F. and Aubier, M. G.: Io-dependent sources of the Jovian decametric emission, Astron. Astrophys., 150, 139-150, 1985.

Godunov, S. K. and Ryabenkii, V. S.: Difference Schemes: an Introduction to the Underlying Theory, 489, Amsterdam: NorthHolland, 1987.

Kopp, A.: Modification of the electrodynamic interaction between Jupiter and Io due to mass loading effects, J. Geophys. Res., 101, A11, 24 943-24 954, 1996.

Landau, L. D. and Lifshitz, E. M.: Electodynamics of continious media, Pergamon Press, Oxford, 1960.

Linker, J. A., Kivelson, M. G., and Walker, R. J.: A threedimensional MHD simulation of plasma flow past Io, J. Geophys. Res., 96, A12, 21 037-21 053, 1991.

Mei, Y., Thorne, R. M., and Bagenal, F.: Analytic model for the density distribution in the Io plasma torus, J. Geophys. Res., 100, A2, 1823-1828, 1995.

Menietti, J. D. and Curran, D. B.: Instantaneous Io flux tube as the source of Jovian DAM: Possible second harmonic emissions, J. Geophys. Res., 95, A12, 21 273-21 280, 1990.

Neubauer, F. M.: The sub-Alfvénic interaction of the Galilean satellites with the Jovian magnetosphere, J. Geophys. Res., 103, 9, 19843-19866, 1998.

Pudovkin, M. S. and Semenov, V. S.: Stationary frozen-in coordinate system, Ann. Geophysicae, 33, 429-433, 1977.

Queinnec, J. and Zarka, P.: Io-controlled arcs and Io-Jupiter interaction, J. Geophys. Res., 103, A11, 26 649-26 666, 1998. 